\title{
Generalized Partial Response for Equalized Channels with Rational Spectra
}

\author{
DAVID G. MESSERSCHMITT, MEMBER, IEEE
}

\begin{abstract}
Harashima and Miyakawa [1] and Tomlinson [2] have described a generalized partial response technique which achieves the performance of the decision-feedback equalizer without the error propagation problem. We show here that when the equalized and baud-rate sampled channel assumes the special rational $z$ transform
\end{abstract}

$$
F(Z)=\frac{\sum_{i=0}^{M} g_{i} Z^{i}}{1 / L \sum_{i=0}^{N} l_{i} Z^{i}}
$$

where $l_{0}=L, g_{0}=1$, the $g_{i}$ and $l_{i}$ are integers, and $L$ is a power of 2 , the implementation can assume an especially simple form not requiring the storage of analog samples. The numerator polynomial can be chosen to achieve transmission zeros, as in ordinary partial response, while the denominator can be chosen to reduce the noise enhancement in equalization. This technique results in as much as a doubling of the peak transmitted voltage and, as in ordinary partial response, an increase in the number of received levels.

It is shown that on the $f^{1 / 2}$ channel characteristic of coaxial cable, most of the noise advantage of decision-feedback equalization can be achieved with a moderate number of received levels, and that some of this noise advantage can be traded for a reduced number of received levels. The greatest advantage accrues in multilevel transmission because of the lower peak transmitted voltage penalty.

\section{INTRODUCTION}

$\mathbf{H}$ ARASHIMA and Miyakawa [1] and Tomlinson [2] have independently described a method of data precoding in a pulse-amplitude modulation (PAM) transmitter which eliminates intersymbol interference from past data digits at the receiver without noise enhancement. Modulo arithmetic is employed in the transmitter to limit the peak transmitted power. This technique is essentially a generalization of partial response (PR) [3]-[7] to channels with noninteger valued overall sampled impulse responses [1].

The implementation of $\mathrm{PR}$ is relatively simple, with the storage of a small finite number of integer-valued past transmitted data and a modulo addition. The generalized partial response (GPR) technique, on the other hand, requires the storage of a number of noninteger valued (analog) past transmitted precoded samples. Of course these analog samples can also be quantized and stored as binary. numbers, but the resultant memory is much larger than that required for $P R$.

Paper approved by the Associate Editor for Data Communication Systems of the IEEE Communications Society for publication without oral presentation. Manuscript received January 24, 1975; revised May 5,1975 .

The author is with Bell Laboratories, Holmdel, N. J. 07733
In this paper we will show that when the equalized and sampled linear channel has only poles and no zeros (which we term an autoregressive equalized channel in analogy to random process terminology) the GPR implementation can be reduced to 1) the storage of a finite number of past data digits (which are generally binary) equal to the number of channel poles, 2) the storage of an equal number of bounded integers which summarizes the state of the channel, 3) the generation of an analog transmitted sample which is a simple transformation of these stored values, and 4) an updating of the stored integers which can be accomplished by read-only memory. This implementation, which avoids the storage of analog samples or quantized versions of analog samples, takes advantage of the Markov properties of the channel.

The importance of the all-pole equalized channel is that the unequalized channel characteristic can generally be fairly closely approximated with relatively few poles, with the result that the noise enhancement incurred in the process of easily implemented equalization is minimal. The use of PR, on the other hand, is usually motivated by a desire to place zeros in the overall response, usually at de or at the half-baud rate, although it has also been shown to have a noise advantage on channels with a fast roll-off [8], [9]. In order to accomplish transmission zeros in the context of the autoregressive GPR it will be shown that the system can' be combined with PR to achieve transmission zeros.

While our realization of GPR simplifies the transmitter structure, it shares with PR and previously described GPR schemes an increase in the number of received levels, complicating the implementation of the receiver.

As will be shown later, the ultimate noise performance of GPR is essentially that of the decision-feedback equalizer [10], but without the error-propagation problems. It has been shown that the potential improvement in performance over linear equalization for decision-feedback equalization is substantial on some practical channels [11] when error propagation is ignored. In some instances it has been shown that error propagation is not a serious impairment [12], [13]. This includes the coaxial cable example considered later in this paper.

\section{DESCRIPTION OF GENERALIZED PARTIAL RESPONSE}

This section will give a brief tutorial explanation of the GPR technique [1], [2]. As a starting point, consider the linear system of Fig. 1(a), which consists of trans- 


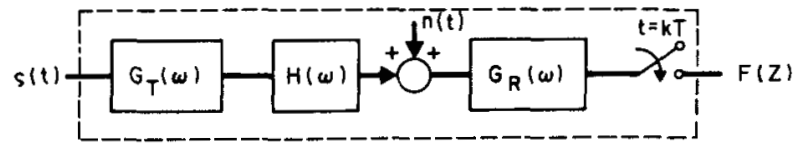

(a)

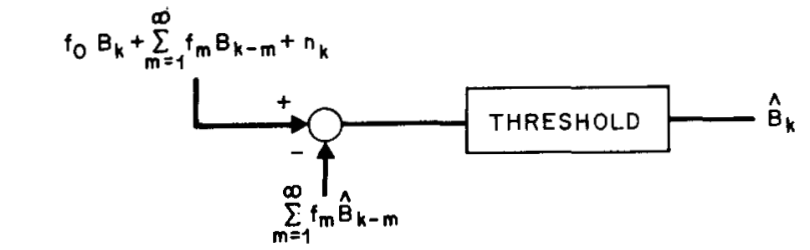

(b)

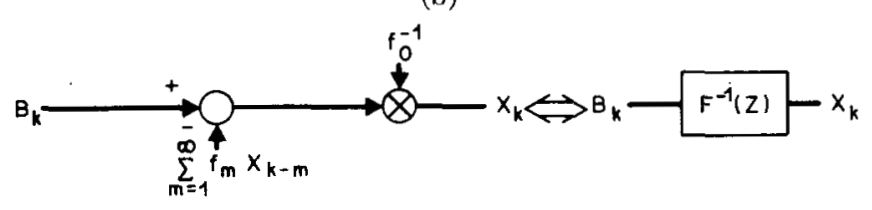

(c)

Fig. 1. Decision-feedback and transmitter prefiltering. (a) Equivalent equalized and sampled linear channel. (b) Tail cancellation by decision-feedback. (c) Tail cancellation by prefiltering.

mitter filter $G_{T}(\omega)$, receiver filter $G_{R}(\omega)$, channel transfer function $H(\omega)$, additive noise $n(t)$, and a baud-rate sampler which samples at $t=k T$, where $T$ is the baud interval. Assume that when the system input is excited by a unit impulse, the output sequence in the absence of noise has $z$ transform $F(Z)$. Assume further that $G_{T}(\omega)$ and $G_{R}(\omega)$ have been chosen such that $F(Z)$ is strictly causal (conditions on $H(\omega)$ for this to be possible are given in $[14],[15])$,

$$
F(Z)=\sum_{m=0}^{\infty} f_{m} Z^{m} .
$$

Therefore, if we transmit a sequence of data digits $\left\{B_{k}\right\}$, the reception is

$$
r_{k}=f_{0} B_{k}+\sum_{m=1}^{\infty} f_{m} B_{k-m}+n_{k}
$$

where $n_{k}$ is the additive noise. The summation term in (2) is an undesirable intersymbol interference which we would like to eliminate prior to a decision on $B_{k}$. One method of accomplishing this is by choosing $G_{R}(\omega)$ so that $f_{m}=0$, $m \geq 1$, but this can only be done at the expense of some noise enhancement: Another approach [10] is to use past decisions to subtract out this interference prior to the receiver decision threshold [Fig. 1(b)], but a penalty in error rate due to error propagation must be accepted.

This error propagation problem is eliminated by the data prefiltering technique of Fig. 1(c). In this method, instead of transmitting $B_{k}$, an analog level $x_{k}$ is substituted. The residual intersymbol interference is subtracted from $B_{k}$ to determine $x_{k}$. This is equivalent to putting a filter $F^{-1}(Z)$ in the transmitter; it achieves the same performance as the decision-feedback method but without

I Note that linear equalization in the receiver to eliminate the intersymbol interference also corresponds to a $F^{-1}(Z)$ filter, but unlike that at the transmitter, enhances the noise. the error propagation problem. However, the peak transmitted signal can be very large (or even infinite, if the filter is unstable).

GPR modifies the prefiltering method just described to limit the peak transmitted power. We will initially discuss the binary case for simplicity, and generalize to multilevel transmission later. Let the data assume the two values $B_{k}= \pm 1$ (binary antipodal signaling). The received signal in the absence of noise and intersymbol interference then assumes one of two values, as illustrated in Fig. 2(a) ( $\pm f_{0}$, where $f_{0}=1$ is assumed). For GPR the received levels are modified to those of Fig. 2(b). Data $B_{k}=1$ now correspond to any of the received levels $(1+\alpha+n \beta)$ where $n$ is an integer, and similarly data $B_{k}=-1$ correspond to any of the received levels $(-1+$ $\alpha+n \beta)$. We list below some considerations relative to the received levels of Fig. $2(\mathrm{~b})$.

1) The motivation for using these levels is to limit the peak transmitted voltage to $\beta / 2$, as will be seen shortly.

2) The constant $\alpha$ is an arbitrary offset which does not impact the error probability. Unless otherwise stated, we assume that $\alpha=0$.

3) The constant $\beta$, which determines the peak transmitted power, should be greater than two so that the input data digit can be inferred from the received level in the absence of noise. The choice $\beta=4$ is the most reasonable, since it results in uniformly spaced received levels.

4) In making a decision on the basis of the received levels of Fig. 2(b), an error rate penalty relative to the format of Fig. 2 (a) is necessarily incurred. For $\beta=4$ the error rate is approximately doubled, and the penalty decreases rapidly as $\beta$ increases from there. Specifically, for Fig. 2(a),

$$
P_{e}=Q\left(\frac{1}{\sigma}\right)
$$

and for Fig. 2(b),

$$
P_{e} \cong 1-Q\left(\frac{-1}{\sigma}\right)+Q\left(\frac{\beta-2}{2 \sigma}\right)
$$

where

$$
Q(x)=\frac{1}{(2 \pi)^{1 / 2}} \int_{x}^{\infty} e^{-y^{2} / 2} d y
$$

and additive Gaussian noise with variance $\sigma^{2}$ has been assumed, as have decision thresholds halfway between received signal levels.

What is the advantage of the received signal format of Fig. 2(b)? Consider the situation from the point of view of the transmitter. It has stored all the values of all the past transmitted precoded data samples, and thus knows the value of the intersymbol interference the receiver will experience; that is, the transmitter knows what the received sample will be if the transmitter sends nothing 


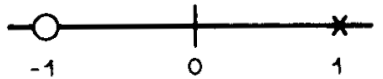

(a)

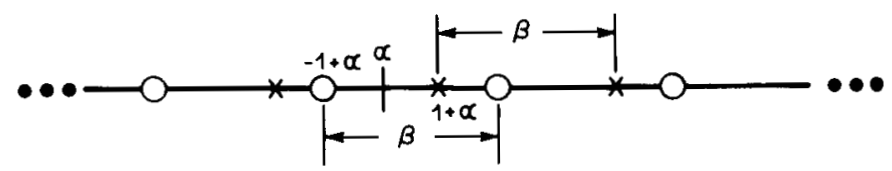

(b)

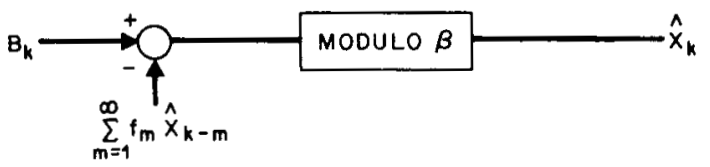

(c)

Fig. 2. Generalized partial response. (a) Binary polar received levels. (b) Generalized partial response received levels. (c) GPR transinitter precoding.

$\left(x_{k}=0\right)$. If the current data are $B_{k}=1$, the transmitter needs to send only a large enough signal to drive the received sample to the nearest point $(1+\alpha+n \beta)$. Thus the largest $x_{k}$ which is ever required is

$$
\left|x_{k}\right| \leq \beta / 2 \text {. }
$$

The peak transmitted power has now been reduced to manageable proportions. For instance, if $\beta=4$, the peak voltage is now potentially double $(6 \mathrm{~dB})$ that required? in the absence of precoding.

A transmitter organization which accomplishes the scheme just described was invented independently by Harashima and Miyakawa [1] and Tomlinson [2]. The received sample in the absence of noise is just (2) with $n_{k}=0$. In view of Fig. $2(\mathrm{~b})$, the received sample is also

$$
r_{k}=B_{k}+\alpha+L_{k} \beta
$$

for some integer $L_{k}$. Equating (2) (with $n_{k}=0$ and $\left\{B_{k}\right\}$ replaced by $\left.\left\{x_{k}\right\}\right)$ with $(7)$, we get the precoded sample (assuming $f_{0}=1$ )

$$
x_{k}=\alpha+L_{k} \beta+B_{k}-\sum_{m=1}^{\infty} f_{m} x_{k-m}
$$

where $L_{k}$ is chosen to satisfy (6) (which is merely a modulo $\beta$ operation). This leads to the transmitter organization of Fig. 2(c) (for $\alpha=0$ ). Note that when the $\left\{f_{m}\right\}$ are summable, $r_{k}$ is bounded by

$$
\left|r_{k}\right| \leq \sum_{m=0}^{\infty}\left|f_{m} x_{k-m}\right| \leq \frac{\beta}{2} \sum_{m=0}^{\infty}\left|f_{m}\right|
$$

and the number of received levels is finite, as required by receiver implementation. The bound of $(9)$ is quite weak, and in practice $r_{k}$ would remain much smaller.

From the implementation point of view, the multilevel received data and transmitter modulo operation represent obstacles. Particularly troublesome, however, is the necessity of storing analog values of previous precoded data samples. This should be compared with the decision feedback of Fig. 1(b) (where past binary decisions are stored) and $\mathrm{PR}^{2}$ (where past integer valued precoded data samples are stored). It will be shown in Section III that if the equalized channel response $F(Z)$ is chosen to be autoregressive (rational in $Z$ with no zeros) the storage of analog samples can be completely avoided. It will then be shown that zeros of $F(Z)$ can also be tolerated as long as the numerator polynomial has integer-valued coefficients and the denominator coefficients assume a special form.

\section{A. GPR for Multilevel Data}

GPR as described in (8) extends straightforwardly to multilevel input data. In fact, for this case a much smaller peak power penalty at a given level of performance can be accommodated. In particular, when $B_{k}$ assumes one of $L$ levels equally spaced on the interval $[-1,1]$, the spacing between levels is $2 /(L-1)$. This same equal level spacing at the receiver can be maintained with $\beta=2+2 /(L-1)$, with a peak transmitted voltage bounded by $\beta / 2=L /(L-1)$. The resultant upper bound on the increase in peak transmitted power is summarized below.

\begin{tabular}{rl}
\multicolumn{1}{c}{ (decibels) } \\
2 & 6.0 \\
4 & 2.5 \\
8 & 1.16 \\
16 & 0.56
\end{tabular}

We see that this penalty decreases rapidly with $L$.

The average transmitted power is also of concern. J. E. Mazo and J. Salz have shown in an unpublished memorandum that when $B_{k}$ assumes one of the $L$ levels equally spaced on $[-1,1]$, the average output power for GPR is bounded by

$$
\frac{1}{3}\left(\frac{L+1}{L-1}\right) \leq P \leq \frac{1}{3}\left(\frac{L+1}{L-1}\right)+\frac{1}{(L-1)^{2}}
$$

where independent equally likely data are assumed. The lower bound in (10) is just the power in the absence of GPR. The upper bound on the penalty in average power in GPR decreases with $L$.

In the sequel, we will concentrate on the simple case of binary transmission ( $L=2$ ). However, the fact that these upper bounds on peak and average transmitted power decrease rapidly with increasing $L$ should be kept in mind, since they suggest that GPR becomes more attractive as $L$ increases in either a peak or average power limited environment. Of course, it should also be kept in

${ }^{2}$ It is readily shown that GPR reduces to $\mathrm{PR}$ when $f_{m}=0$ for $m>$ some $M$, the remaining $f_{m}$ are integers, and $\alpha=-1$. There is then no peak power penalty at the transmitter. 
mind that implementation difficulties in resolving the multilevel received levels compound as $L$ increases.

\section{GPR FOR AUTOREGRESSIVE EQUALIZED CHANNELS}

Let $F(Z)$ be of the autoregressive form

$$
\begin{aligned}
F(Z) & =\frac{1}{\sum_{i=0}^{N} d_{i} Z^{i}}, \quad d_{0}=1 \\
& =\frac{1}{\prod_{i=1}^{N}\left(1-e_{i} Z\right)}, \quad\left|e_{i}\right|<1 .
\end{aligned}
$$

The $\left\{e_{i}\right\}$ will in general be complex numbers which occur in conjugate pairs. Before considering GPR for this response, first consider the non-GPR prefiltering method of Fig. $1(\mathrm{c})$. Since $F^{-1}(Z)$ is simply a polynomial in $Z$,

$$
x_{k}=\sum_{i=0}^{N} d_{i} B_{k-i} \text {. }
$$

This simplified implementation does not involve storing analog samples, but rather only $N-1$ past data digits. In addition, we note that if the data are binary, for instance, $x_{k}$ can only assume one of $2^{N+1}$ values, and the peak transmitted power is strictly bounded in well-defined fashion.

In order to implement the GPR for this channel we cannot simply reduce (12) modulo $\beta$, since the receiver's intersymbol interference is determined by the past precoded data (which was itself reduced modulo $\beta$ ) and the

fact that the $\left\{d_{i}\right\}$ may not be integers. However, we will now show that the transmitter can keep track of the intersymbol interference at the receiver without storing the past precoded samples explicitly.

Assume that the received sample $r_{k}$ is the standard GPR level of $(7)^{3}$ and that the transmitted precoded data sample is $x_{k}$. Then

$$
\begin{aligned}
R(Z) & =F(Z) X(Z) \\
& =B(Z)+\beta L(Z) .
\end{aligned}
$$

in obvious notation. Taking the inverse $z$ transform,

$$
x_{k}=B_{k}+\beta L_{k}+\sum_{i=1}^{N} d_{i}\left(B_{k-i}+\beta L_{k-i}\right) .
$$

At the time that $x_{k}$ is determined, $L_{k-i}, 1 \leq i \leq N$ have already been specified by previous transmitted samples. However, we are free to choose $L_{k}$, and the obvious choice is the one which forces (6). Note again that for nonintegers $\left\{d_{i}\right\}$ (14) does not reduce to (12), even on a modulo $\beta$ basis. The transmitter must store $N$ past data

${ }^{3}$ We assume that $\alpha=0$ in the sequel. digits $\left(B_{k-1}, \cdots, B_{k-N}\right)$ and $N$ past values of $L_{k},\left(L_{k-1}, \cdots\right.$, $\left.L_{k-N}\right)$. It then determines $L_{k}$ and the precoded data sample $x_{k}$ by the standard modulo $\beta$ operation using its knowledge of the $\left\{d_{i}\right\}$.

Implementation becomes especially easy in the binary case when the coefficients $\left\{d_{i}\right\}$ are chosen judiciously. Let there be integers $L, l_{i}$ such that

$$
d_{i}=\frac{l_{i}}{L}, \quad 0 \leq i \leq N, \quad l_{0}=\dot{L}
$$

(that is, let $\left\{d_{i}\right\}$ be a set of rational numbers with common denominator $L$ ). Then (14) becomes

$$
x_{k}=\frac{1}{L} \sum_{i=0}^{N} l_{i}\left(B_{k-i}+\beta L_{k-i}\right) \text {. }
$$

All the factors in $(16)$, with the exception of $(1 / \dot{L})$, are integers as long as $\beta$ is an integer. ${ }^{4}$ The transmitter implementation is then reduced to integer arithmetic, and a modulo $\beta L$ operation (which is simple when $\beta$ is an integer). The transmitter must then generate one of $\beta L$ equally spaced levels, which is easily accomplished with a uniform $\mathrm{D} / \mathrm{A}$ converter. When $L$ is a power of 2 , the implementation is particularly simple: if the transmitter is implemented with binary arithmetic, and the proper word length is chosen for the representation of the results of arithmetic operations, the modulo operation is implicitly and automatically performed by any overflow. The generation of a number of equally spaced analog levels which is a power of 2 is also particularly convenient.

It is critical to the implementation of both transmitter and receiver that $L_{k}$ be an integer which is strictly.bounded. It is apparent that the sequence of precoded data samples will be identical for our scheme and the one described in Section II (the precoded samples are unique) so that the bound on $r_{k}$ (and hence $L_{k}$ ) of (9) still applies. Thus, we can assert that $L_{k}$ is bounded as long as $\left\{f_{m}\right\}$ is absolutely summable, which will always be the case for the $F(Z)$ of (11) (since there are no poles on the unit circle). Finally, we mention in passing that for $\beta=4, \alpha=0$, and

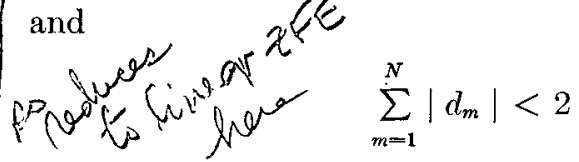

the modulo $\beta$ operations in transmitter and receiver are not necessary since the samples of (12) are automatically less than $\beta / 2$. In particular, (17) is always satisfied for $N=1$.

\section{A. Accommodation of Transmission Zeros}

In many applications it is important to have transmission zeros in the overall equalized channel response. Such is the case, for instance, whenever the actual channel medium has no transmission at a certain frequency (most

\footnotetext{
${ }^{4}$ Similarly when $\beta$ is rational, as in the multilevel case of Section II-A, (16) can be placed over a common denominator which is $L$ times the denominator of $\beta$.
} 


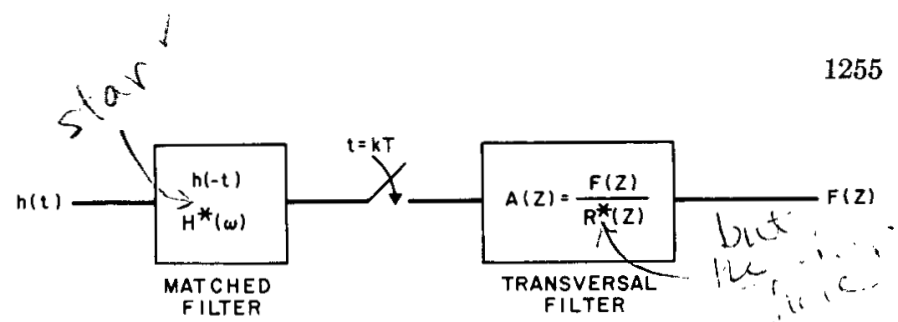

Fig. 3. Optimum equalizer for obtaining response $F(Z)$. signaling or adding a decision-feedback loop into the receiver. However, we will concentrate here in incorporating $\mathrm{PR}$ into our scheme. Let the equalized response have the form

$$
F(Z)=\frac{\sum_{i=0}^{M} g_{i} Z^{i}}{\frac{1}{L} \sum_{i=0}^{N} l_{i} Z^{i}}
$$

where $g_{i}$ and $l_{i}$ are integers and $l_{0}=L, g_{0}=1$. Then

$$
x_{k}=\frac{1}{L} \sum_{i=0}^{N} l_{i}\left(B_{k-i}+\beta L_{k-i}\right)-\sum_{i=1}^{M} g_{i} x_{k-i}
$$

and as before $L_{k}$ can be chosen to satisfy (6). However, we claim that when $\beta$ is an integer, $x_{k}$ assumes the form

$$
x_{k}=\frac{m_{k}}{L}
$$

with integer $m_{k}$. To see this substitute (20) in (19) to get

$$
m_{k}=\sum_{i=0}^{N} l_{i}\left(B_{k-i}+\beta L_{k-i}\right)-\sum_{i=1}^{M} g_{i} m_{k-i}
$$

where all quantities are integers. The transmitter must now store the quantities $\left(B_{k-i}, L_{k-i}\right), 1 \leq i \leq N$ and $m_{k-i}, 1 \leq i \leq M$.

\section{CHOICE OF THE EQUALIZED CHANNEL}

When an overall response of the form of (18) is chosen, the channel output must be equalized to achieve that sampled response. It is readily shown by the standard variational argument [17] that the minimum noise enhancement is achieved by the matched-filter/transversalfilter of Fig. 3, where

$$
\sum_{k} x_{k} h(t-k T)+n(t)
$$

is the receiver input, and $n(t)$ is white Gaussian noise with double-sided spectral density $N_{0} / 2$. Further, we define

$$
\begin{gathered}
R(Z)=\sum_{k} R_{k} Z^{k} \\
R[\exp (j \omega T)]=\frac{1}{T} \sum_{k}\left|H\left(\omega+k \frac{2 \pi}{T}\right)\right|^{2} \\
R_{k}=\int_{-\infty}^{\infty} h(t) h(t-k T) d t
\end{gathered}
$$

as can be found in [15]. The noise spectrum at the sampled matched filter output is $N_{0} / 2 R(Z)$, the noise spectrum at the decision threshold input is $N_{0} F(Z) F\left(Z^{-1}\right) / 2 R(Z)$, and hence the noise variance at the threshold input is

$$
\sigma^{2}=\frac{N_{0}}{4 \pi j} \int \frac{F(Z) F\left(Z^{-1}\right)}{R(Z)} \frac{d Z}{Z}
$$

where contour integration is around the unit circle. The equalized response $F(Z)$ should be chosen to minimize $(26)$

If the solution is not constrained to be autoregressive, (26) is minimized by the choice

$$
F(Z)=C(Z) / C(0)
$$

where

$$
\begin{gathered}
R(Z)=C(Z) C\left(Z^{-1}\right) \\
C(Z) \neq \mathbf{0}, \quad|Z| \leq \mathbf{1}
\end{gathered}
$$

is the unique causal minimum phase factorization of $R(Z)$. The equalized response is then that of the optimum decision-feedback equalizer, for which a "closed form" expression for $\sigma^{2}$ is available [14], [15].

When $\vec{C}(\bar{Z})$ is autoregressive, $F(Z)$ will automatically minimization of (26) for autoregressive $F(Z)$ with $F(0)=1$ does not appear possible, and numerical solution is necessary.

A frequent criticism of transmitter precoding schemes is that there is no possibility of adapting the transmitter to a time-varying channel without a separate feedback channel. However, this criticism is not justified when, as on coaxial cable channels, the channel variations are small enough that a single equalized $F(Z)$ will give a substantial noise advantage over the entire ensemble of channels. In in the sense that it tries to achieve the equalized response $F(Z)$ assumed by the transmitter. In this way, the technique can be applied to advantage to some time-varying channels.

\section{DETERMINATION OF RANGE OF RECEIVED LEVELS}

The determination of the range of $L_{k}$ has important implications to receiver complexity, gain stabilization, and timing recovery. If we can assert that $\left|\bar{L}_{k}\right| \leq \bar{L}_{\max }$, then the potential number of received levels is $\left(2 L_{\max }+1\right)$ times the number of data levels. In this section we will describe an efficient algorithm which has been programmed on the computer and which determines the range of $L_{k}$ for a given equalized channel. The method as we will be autoregressive. Otherwise, an analytic solution of the that case, the receiver equalizer can be made adaptive 
describe it is applicable to the pure autoregressive case only, but it can be generalized in straightforward fashion to the transmission zero case of (18).

It was established by (9) that the range of received levels is finite. However, this bound is extremely weak, as the following example will illustrate. Let $F(Z)=$ $(1-A Z)^{-1}$, in which case

$$
x_{k}=B_{k}-\dot{A} B_{k-1}
$$

the modulo operation is not required as long as $|A|<1$, and

$$
\left|x_{k}\right| \leq 1+A
$$

for $A>0$. By the method of (9), we have

$$
\left|r_{k}\right|=\left|\sum_{m=0}^{\infty} A^{m} x_{k-m}\right| \leq \frac{1+A}{1-A} .
$$

However, we know that in this case $r_{k}= \pm 1$, and the bound of (29) becomes progressively weaker as $A \rightarrow 1$.

Determining the actual range of $L_{k}$ is quite straightforward, at least in principle. Define the state of the system at time $k$ as

$$
S_{k}=\left(L_{k-i}, B_{k-i}, 1 \leq i \leq N\right) .
$$

Then, based on (6) and (13), the new state $S_{k+1}$ is a well defined function of $S_{k}$ and $B_{k}$,

$$
S_{k+1}=f\left(B_{k}, S_{k}\right)
$$

We can divide the states into mutually exclusive groups corresponding to each possible $\left(B_{k-1}, \cdots, B_{k-N}\right)$, as illustrated for the binary case with $N=2$ by the four boxes in Fig. 4(a). When the state is updated, depending on which of the two values of $B_{k}$ occurs, a state in one of the two new boxes will result. The movement of states between boxes as a function of $B_{k}$ is shown in Fig. 4(a). The motion of the system is also determined by the initial condition, which we specify as

$$
B_{k}=L_{k}=0, \quad k<0 .
$$

Based on (32), the state $S_{N}$ is determined solely by the data sequence $\left(B_{N-1}, \cdots, B_{0}\right)$ and not by any past history of the system; that is, there is only one state $S_{N}$ for each of the boxes in Fig. 4(a). These initial states are shown in Fig. 4(b), and marked with a "zero" to indicate that they are initial conditions.

Now, as illustrated in Fig. 4(c), for each initial "zero" state there are two new states $S_{N+1}$ generated in boxes determined by Fig. 4(a). One or both of these new states may, in fact, be an already existing state, as illustrated by the transition from the "zero" state in the lower right box to the "zero" state in the lower left box corresponding to $B_{k}=1$. All the truly new states are marked by a "one" to indicate the first iteration. At the next iteration, illustrated in Fig. 4(d), transitions from all the states marked "one" are determined. In this case, all the transitions end in already existing states. This must happen eventually

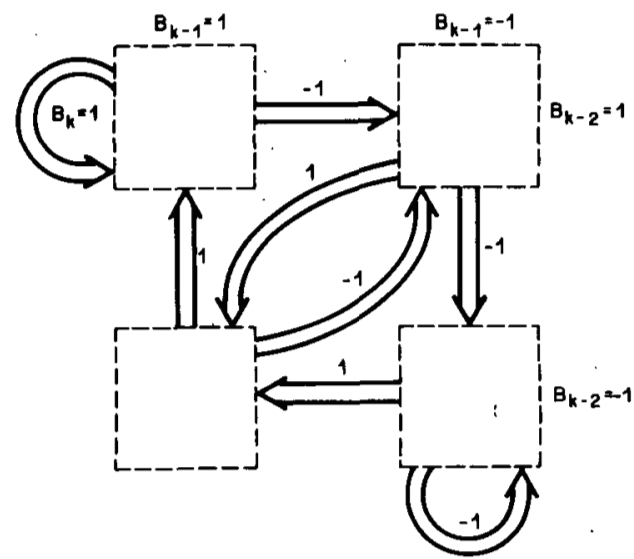

(a)
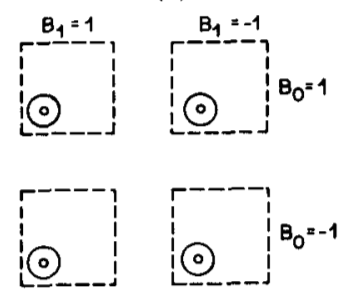

(b)

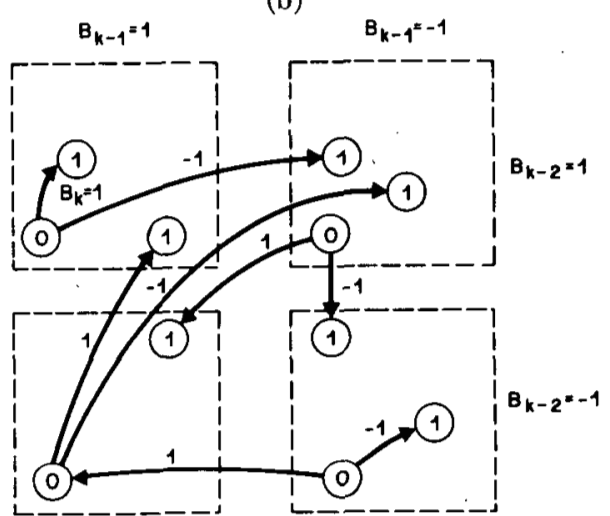

(c)

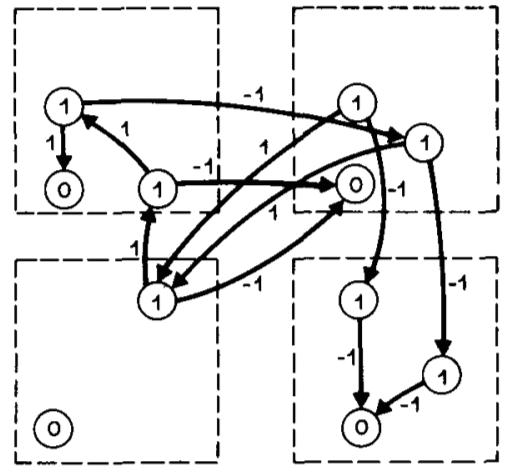

(d)

Fig. 4. Determining number of received levels. (a) Movement of states when $B_{k}=1$ or $B_{k}=-1$. (b) Initial states. (c) Generation of new states. (d) Termination of states.

(although usually not at the second iteration!), since the total number of states is finite, and we cannot continue to generate new states indefinitely. The result is a complete and minimal set of states for the system, and the largest $L_{k}$ can be determined accordingly.

The extension of this Markov chain approach to the system with transmission zeros in (21) is possible, but 
only at the expense of a far greater number of states. Since $L_{k}$ is chosen such that

$$
-2<\left|\frac{m_{k}}{L}\right| \leq 2
$$

for $\beta=4$, it follows that

$$
-2 L+1 \leq m_{k} \leq 2 L \text {. }
$$

Thus the number of possible sequences $\left(m_{k-1}, \cdots, m_{k-M}\right)$ is finite, and the state of (30) can be augmented to

$$
S_{k}=\left(L_{k-i}, B_{k-i}, 1 \leq i \leq N, m_{k-i}, 1 \leq i \leq M\right) .
$$

The number of states remains finite and (31) remains valid, so that the remainder of the analysis proceeds without major modification.

It should also be straightforward, although we have not attempted to do so, to determine exactly the peak and average transmitted power for our autoregressive GPR case by exploiting the finite state structure just described.

\section{APPLICATION TO THE $\sqrt{f}$ CHANNEL}

In this section we will determine the reduction in noise enhancement which can be obtained on the $\sqrt{f}$ channel characteristic of coaxial cables and some wire pairs. The frequency response is given by [11]

$$
|H(\omega)|^{2}=2 \pi K^{2} R_{0} \exp \left(-2 K \omega^{1 / 2}\right)
$$

where $R_{0}$ is the energy in an isolated pulse, $K$ is a constant / proportional to the line length, and transmitted impulses - are assumed. If we define $\gamma$ to be the loss of the line at the half-baud rate in decibels, then

$$
K=\left(\frac{T}{\pi}\right)^{1 / 2} \frac{\gamma}{20 \log _{10} e}
$$

\section{A. Optimum Pole Placement}

The minimization of (26) was accomplished for an autoregressive $F(Z)$ using an iterative optimization program. The program was initially run for $\gamma=60$ and with one to four complex poles constrained to lie outside the unit disk; the result in each case was a set of real and equal poles. While this may be a local rather than global minimum, the performance figures to follow indicate that it is a good solution in the sense that the maximum attainable improvement is approached rapidly. The resulting equalized channel is given by

$$
F(Z)=(1-A Z)^{-N}
$$

where $A^{-1}$ is the pole location,

$$
? \quad-1 \leq A \leq 1 \text {. }
$$

A matched filter output has noise variance $N_{0} / 2 R_{0}$ (when the signal is normalized to unit height), so that the noise penalty relative to the matched filter bound for an isolated pulse is given by

$$
10 \log \frac{2 R_{0} \sigma^{2}}{N_{0}}
$$

where $\sigma^{2}$ is given by $(26)$. When $N=0$ (the zero forcing equalizer case), (37) is $30.3 \mathrm{~dB}$ for $\gamma=60 \mathrm{~dB}$. The minimum value of (37) which is attainable by GPR is the value for the decision-feedback equalizer, and is $19.9 \mathrm{~dB}$ when $\gamma=60 \mathrm{~dB}$ [11]. Thus, there is a potential $10 \mathrm{~dB}$ performance advantage to be gained by GPR.

When the $A$ of (36) was chosen optimally, it was found that the resulting $L_{\max }$ was excessive. Therefore, it is necessary to sacrifice some noise advantage to limit the number of received levels. The numerical results are shown in Fig. 5, where the range of received levels was determined by programming the algorithm of Section $\mathrm{V}$. The regions of fixed $L_{\mathrm{m} \text { ax }}$ are delimited by dashed lines.

It is also interesting to compare GPR with Class I PR [5] which has been shown to have a noise advantage ${ }^{5}$ on coaxial cable [8], [9]. The attractive feature of Class I PR is that it has no peak or average transmitted power penalty. As shown on Fig. 5, Class I PR has a $5 \mathrm{~dB}$ advantage over zero forcing equalization (we found Class II to have a virtually identical advantage).

The results of Fig. 5 are summarized below.

1) A substantial (5 dB) advantage is obtained by GPR without any increase in the number of received levels $\left(L_{\mathrm{max}}=0\right)$. However, this same advantage can be obtained with Class I PR, with no transmitted power penalty, and three received levels for binary transmission.

2) For $L_{\max } \geq 1$, the advantage of $\mathrm{GP} \overline{\mathrm{R}}$ increases with the number of poles $(N)$, which is related to the complexity of the transmitter. VS, icis:

3) About 9 of the potential $10 \mathrm{~dB}$ advantage can be achieved with $L_{\max }=1$ or 2 . However, if GPR is penalized for its maximum possible increase in peak transmitted power, it has an advantage over Class I PR only for multilevel (but not binary) transmission. ${ }^{6}$

Finally, the reader is cautioned that those results apply only to coaxial cable with $\gamma=60 \mathrm{~dB}$. In particular, on channels with a more modest roll-off with frequency, Class I PR often incurs a noise penalty (not advantage).

\section{CONCLUSIONS}

We have described a modification of GPR which is valid for an overall equalized channel with rational spectrum and which eliminates the necessity of storing analog samples in the transmitter. This technique can come close to achieving the improvement in noise penalty of the decision-feedback equalizer without the error propagation problem of that receiver. The inevitable price that is paid for this benefit is 1) a potential doubling of the peak transmitted voltage in the binary case and 2) (usually) an increase in the number of received levels. The technique can be applied to channels with a moderate amount

\footnotetext{
5 This noise advantage for optimum receiver equalization is easily evaluated by letting $F(Z)=1+Z$ in (26).

- Harashima and Miyakawa [16] have previously shown that on coaxial cable a large number of transmitting levels is optimum with GPR.
} 


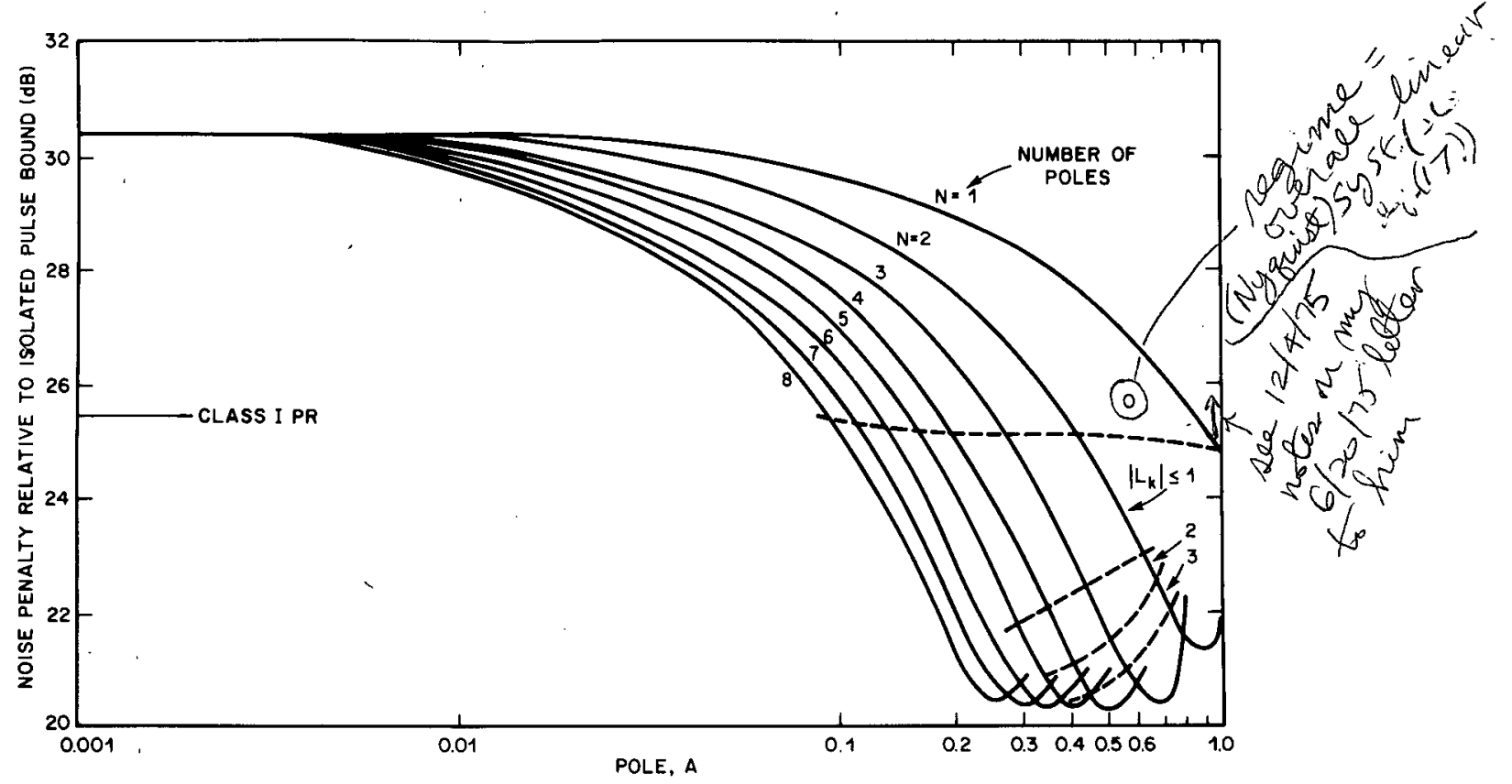

Fig. 5. Noise penalty versus pole position, $N$ equal real poles, $\gamma=60 \mathrm{~dB}$.

of time variation by making the receiver equalization adaptive.

Application to a coaxial cable channel reveals that in this case GPR can yield a substantial advantage over zero-forcing equalization exclusively at the receiver, but that if GPR is penalized for the upper bound on its increase in peak transmitted power, it is more attractive than Class I PR only for multilevel (but not binary) data. Decision-feedback equalization remains an attractive alternative, since it incurs no increase in transmitted power or number of received decision levels, and for random data its error propagation can be controlled by limiting the number of feedback taps [13].

\section{ACKNOWLEDGMEN'T}

The author is indebted to Dr. R. Price for many valuable comments on the manuscript.

\section{REFERENCES}

[1] H. Harashima and H. Miyakawa, "Matched-transmission technique for channels with intersymbol interference," IEEE Trans. Commun. (Special Issue on Communications in Japan), vol. COM-20, pp. 774-780, Aug. 1972.

[2] M. Tomlinson, "New automatic equalizer employing modulo arithmetic," Electron. Lett., vol. 7, Mar. 1971.

[3] A. Lender, "The duobinary technique for high-speed data transmission," IEEE Trans. Commun. Electron., vol. 82, pp. 214-218, May 1963.

[4] "Correlative level coding for binary-data transmission," IEE' Spectrum, vol. 3, pp. 104-115, Feb. 1966.

[5] E. R. Kretzmer," "Generalization of a technique for binary data communication," IEEE Trans. Commun. Technol. (Concise Papers), vol. COM-14, pp. 67-68, Feb. 1966.

[6] A. M. Gerrish and R. D. Howson, "Multilevel partial response signaling," presented at the 1967 Int. Conf. Commun., Minneapolis, Minn., June 1967.

[7] D. W. Tufts and C. V. Ramamoorthy, "Modulo- $m$ linear sequential circuits, partial response signaling formats, and signal-flow graphs," Div. Eng. and Appl. 'Physics, Harvard Univ., Cambridge, Mass., Tech. Rep. 519 (AD-650958), Feb. 1967.

[8] T. Ericson, "Optimum PAM-filters and some applications," Telecommunication Theory, Royal Inst. Technol., Stockholm, Sweden, Tech. Rep. 50, pp. 27-29, May 1972.

[9] S. A. Fredricsson, "Joint optimization of transmitter and re- ceiving filters in digital PAM-systems with a Viterbi detector," Telecommunication Theory, Royal Inst. Technol., Stockholm, Sweden, Tech. Rep. 89, pp. 20-21, 37, Oct. 1974.

[10] L. A. McColl, "Signaling method and apparatus," U. S. Patent 2056284 , Oct. 1936.

[11] D. G. Messerschmitt, "A geometric theory of intersymbol interference-Part II: Performance of the maximum likelihood detector," Bell Syst. Tech. J., vol. 52, pp. 1521-1539, Nov. 1973.

[12] D. L. Duttweiler, J. E. Mazo, and D. G. Messerschmitt, "An upper bound on the error probability in decision-feedback equalization," IEEE Trans. Inform. Theory, vol. IT-20, pp. 490-497, July 1974 .

[13] D. G. Messerschmitt, "Design of a finite impulse response for the Viterbi algorithm and decision-feedback equalizer," presented at the Int. Conf. Commun., Minneapolis, Minn., June 1974 .

[14] R. Price, "Nonlinearly feedback-equalized PAM vs. capacity for noisy filter channels," presented at the 1972 Int. Conf. Commun., Philadelphia, Pa., June 1972.

[15] D. G. Messerschmitt, "A geometric theory of intersymbol interference-Part I: Zero-forcing and decision feedback equalization," Bell Syst. Tech. J., vol. 52, pp. 1483-1519, Nov. 1973.

[16] H. Harashima and H. Miyakawa, "Capacity of channels with matched transmission technique for peak transmitting power limitation," in Nat. Conv. Rec. Inst. Electron. Commun. Eng. Japan, no. 1268, Aug, 1969 (English translation available from

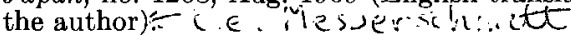

[17] D. D. Falconer and F. R. Magee, Jr., "Adaptive channel memory truncation for likelihood sequence estimation," Bell Syst. Tech. J., vol. 52, pp. 1541-1562, Nov. 1973.

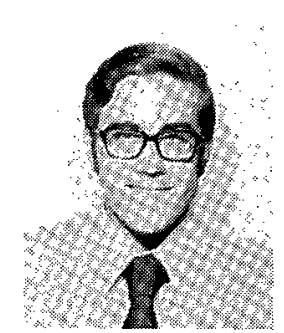

David G. Messerschmitt (S'65-M'68) was born in Denver, Colo., on May 21, 1945. $\mathrm{He}$ received the B.S.E.E. degree from the University of Colorado, Boulder, in 1967, and the M.S. and Ph.D. degrees, from the University of Michigan, Ann Arbor, in 1968 and 1971 , respectively.

Since 1968, he has been with Bell Laboratories, Holmdel, N.J., where he has been studying problems of intersymbol interference and voice coding for digital transmission. He currently supervises a group studying bit-rate reduction techniques.

Dr. Messerschmitt is a member of Eta Kappa Nu, Tau Beta Pi, and Sigma Xi. He is Vice-Chairman of the Jersey Shore Chapter and a member of the Communication Theory Committee of the IEEE Communications Society. 